\title{
Star Formation-Regulated Growth of Black Holes in Protogalactic Spheroids
}

\author{
Andreas Burkert \\ Max-Planck-Institut für Astronomie, Königstuhl 17, \\ D-69117 Heidelberg, \\ Germany \\ Joseph Silk \\ Department of Astrophysics, University of Oxford, NAPL, \\ Keble Road, Oxford OX13RH, \\ United Kingdom
}

Received — 


\begin{abstract}
The observed relation between central black hole mass and spheroid velocity dispersion is interpreted in terms of a self-regulation model that incorporates a viscous Keplerian accretion disk to feed the black hole, embedded in a massive, self-gravitating star forming disk that eventually populates the spheroid. The model leads to a constant ratio between black hole mass and spheroid mass which is equal to the inverse of the critical Reynolds number for the onset of turbulence in the accretion disk surrounding the central black hole. Applying the fundamental plane correlation for spheroids, we find that the black hole mass has a power-law dependence on the spheroid velocity dispersion with a slope in the range of 4-5. We explain the larger scatter in the Magorrian relation with respect to the black hole mass-spheroid velocity dispersion relationship as a result of secular evolution of the spheroid that primarily affects its luminosity and to a much lesser extent its velocity dispersion.
\end{abstract}

Subject headings: galaxies: nuclei - galaxies: kinematics and dynamics - black hole physics 


\section{Introduction}

The remarkable dependence of black hole mass on spheroid velocity dispersion, with low dispersion $(<10 \%)$ about a mean power-law slope between 4 and 5 (Ferrarese \& Merritt 2000, Merritt \& Ferrarese 2000, Gebhardt et al. 2000a,b) merits serious attention by theorists. Several scenarios have recently been proposed to interpret this relation for either value of the slope, but none are entirely convincing (Silk \& Rees 1998, Ostriker 2000, Kauffmann \& Haehnelt 2000, Adams, Graf \& Richstone 2000). Silk \& Rees (1998) had predicted such a dependence in a model that appealed to feedback for quasar outflows on the protogalactic gas reservoir. They found the relation

$$
M_{b h}=\sigma_{s p h}^{5} f_{c o l d} \alpha,
$$

where $f_{\text {cold }}$ is the cold gas fraction and $\alpha$ depends weakly on the presence of an accretion disk (Haehnelt, Natarajan and Rees 1998) and inversely on the ratio of the kinetic outflow energy to Eddington luminosity. This study requires a very fast accretion phase to feed the central black hole. Recent results by Merrifield, Forbes \& Terlevich (2000) provide evidence for a relationship between black hole mass and bulge age and indicate that the black hole formation timescale is longer, of order a Gyr.

A flatter relation was derived from cold dark matter-dominated cosmologies by Kauffmann \& Haehnelt (2000). They assumed that a fixed fraction of the cold gas supply that forms spheroid stars in a merger will feed the central black hole and discussed the importance of feedback. Without feedback their derived scaling relation is $M_{b h} \propto \sigma_{s p h}^{2}$. However incorporating feedback leads to arbitrarily steeper slopes, depending on the choice of the free parameters, a situation which is not very satisfactory. A relatively flat slope was postulated by Ostriker (2000), for a model in which the central black hole formed from self-interacting dark matter, now largely discredited (Gnedin \& Ostriker 2000; Yoshida et al. 2000). Adams, Graf \& Richstone (2000) proposed a new scenario in which the 
central black hole grew during the protogalactic collapse phase by capturing stars on nearly radial orbits. Their model is however questionable for several reasons: Merrifield's result requires black hole growth on a time-scale longer than that of protogalactic collapse, star formation is unlikely to have been highly efficient during this phase, and finally they adopt a characteristic velocity dispersion $\sigma$ to determine the specific angular momentum of the stars that neglects the fundamental plane relation between $\sigma$ and scale radius.

\section{A Model of self-regulated black hole growth}

We propose an alternative model for black hole growth in forming spheroids that is based on the commonly adopted merging scenario for the formation of spheroids. As first proposed by Toomre \& Toomre (1972), spheroids, like ellipticals, formed via major mergers of gas-rich progenitors. These disk galaxies most likely had already formed central black

holes in their bulges that satisfied the Magorrian relation (Magorrian et al. 1998). However the black hole that results from the coalescence of the progenitor black holes during a spiral-spiral merger would fall short of the Magorrian relation by a large factor. A large fraction of its mass must therefore have been accreted during or after the merger event, presumably as gas.

Major merger simulations (Mihos and Hernquist 1996) demonstrate that the gas rapidly settles, on a short dynamical time-scale, into a central, self-gravitating disk. By that time, the progenitor black holes will also have merged in the centre by dynamical friction (Burkert \& Sunyaev 2000). The inner disk has Keplerian rotation as the gravitational potential is dominated by the mass of the black halo. This region looses angular momentum by viscous drag and the resulting gas inflow will feed the central black halo. At the same time, the outer self-gravitating disk part is gravitationally unstable to fragmentation and subsequent star formation. 
Within the framework of our model, we can estimate the critical radius $r_{c r}$ that separates the two regions dominated by accretion and fragmentation and which is determined by the radius out to which the black hole dominates the gravitational potential,

$$
r_{c r}=G M_{b h} / \sigma_{s p h}^{2} .
$$

Here $\sigma_{s p h}$ is the characteristic velocity dispersion of the spheroid and $M_{b h}$ is the black hole mass. The accretion disk is Keplerian with size $r_{d}$ where it rotates at

$$
v_{r o t}^{2}=G M_{b h} r_{d}^{-1} .
$$

Typical time-scales for the gas in the whole inner disk to accrete onto the black hole are given by the viscous drag timescale $t_{v i s}=r_{d}^{2} / \nu$. For a viscosity prescription, we adopt the formulation: $\nu=R_{c r}^{-1} v_{r o t} r_{d}$, where the critical Reynolds number for the onset of turbulence is $R_{c r} \approx 100-1000$ (Duschl, Strittmatter and Biermann 2000). The viscous time can now be written as

$$
t_{v i s}=G M_{b h} R_{c r} \sigma_{s p h}^{-3},
$$

where we have set $r_{d}=r_{c r}$.

The black hole will grow as long as there is a gas supply for the inner disk. This gas reservoir is replenished by viscous inflow from the outer disk into the accretion region and augmented by the increase in the inner disk radius due to the growth in the black hole mass. In the absence of any limiting factor all the gas supply will eventually be accreted onto the black hole. In this case one would expect a large variance in the black hole mass with respect to the local velocity dispersion unless the initial gas fraction of the merger components was relatively fine-tuned. This seems unphysical on theoretical grounds, nor is any relation observed between gas fraction and velocity dispersion.

We propose that star formation in the outer disk provides the self-regulation that limits the mass of the central black hole. Black hole growth saturates due to the competition with 
star formation, which determines the gas fraction in the disk that is available for accretion. Let $M_{g}$ be the total gas mass available for accretion and $M_{*}$ be the mass in stars within the corresponding radius. We can identify the gas fraction $\epsilon$ as $\epsilon=\frac{M_{g}}{M_{d}}$, where $M_{d}=M_{g}+M_{*}$ is the disk mass at a given radius $\mathrm{r}$, to explicitly demonstrate how black hole growth is limited by the gas supply. Black hole growth only occurs during the gas-rich phase of the protospheroid, that is on the characteristic star formation time-scale $\tau_{s f}$.

Whatever gas is in the inner disk within $r_{c r}$ will be accreted onto the black hole without significant star formation, since self-gravity is unimportant. The average growth rate of the black hole can then be estimated by

$$
\dot{M}_{b h}=\epsilon M_{d} t_{v i s}^{-1}
$$

where $M_{d}, \epsilon$ and $t_{v i s}$ are defined at $r_{c r}$. The total gas fraction within $r_{c r}$ will contribute to the black hole mass. We therefore set $M_{g}=\epsilon M_{d}$ equal to the black hole mass $M_{b h}$. This assumes the initial black hole mass to be negligible with regard to the accreted mass. Viscosity (equation 4) then limits the black hole growth rate to

$$
\dot{M}_{b h}=\sigma_{s p h}^{3} R_{c r}^{-1} G^{-1}
$$

The growth continues until star formation exhausts the gas supply, on a time-scale $t_{s f}$, leading to a final black hole mass

$$
M_{b h}=\sigma_{s p h}^{3} R_{c r}^{-1} G^{-1} t_{s f}=1.9 \times 10^{8}\left(\frac{\sigma_{s p h}}{200 \mathrm{~km} \mathrm{~s}^{-1}}\right)^{3}\left(\frac{R_{c r}}{1000}\right)^{-1}\left(\frac{t_{s f}}{10^{8} \mathrm{yr}}\right) \mathrm{M}_{\odot} .
$$

This fits the observed normalisation of the $\left(M_{b h}, \sigma_{s p h}\right)$ relation for reasonable values of the star formation time-scale and the critical Reynolds number for the onset of turbulence.

It is reasonable to assume that molecular clouds form and fragment into stars on a dynamical time-scale. We therefore write the star formation time-scale as $t_{s f}=\eta t_{d y n}=\eta r_{e} \sigma_{s p h}^{-1}$, where $\eta \sim 1$. Hence

$$
M_{b h}=\eta \sigma_{s p h}^{2} R_{c r}^{-1} G^{-1} r_{e}=M_{s p h} \eta R_{c r}^{-1}
$$


This provides a natural explanation of the Magorrian relation. The ratio of black hole mass to spheroid stellar mass is given by a "universal" constant, $\sim R_{c r}^{-1}$, which in principal should be derivable from fundamental theory and should be insensitive to galaxy parameters. It is remarkable that the ratio of two global masses depends basically on a parameter determined by the microphysics of viscous transport of angular momentum. The mass ratio could therefore provide a direct measure for the typical Reynolds number $R_{c r}$ in accretion disks around massive black holes.

The predicted dependence of $M_{b h}$ on $\sigma_{s p h}$ and $r_{e}$ follows the virial theorem expectation $M_{s p h}=r_{e} \sigma_{s p h}^{2} G^{-1}$ for the spheroid. This leads to a fundamental plane-like projection on spheroid parameters for the black hole mass, with a constant offset that is consistent with the Magorrian relation. Note that $r_{e}$ depends on $\sigma_{s p h}$ and on surface brightness: $r_{e} \propto \sigma_{s p h}^{2}$. This follows, for example by combining the Faber-Jackson relation, $M_{s p h} \propto \sigma_{s p h}^{4}$ with the virial theorem. We infer that $M_{b h} \propto \sigma_{s p h}^{4}$, which is in the observed range.

The crucial issue, however, is that of the remarkable reduction in dispersion for the relation between $M_{b h}$ and $\sigma_{s p h}$ relative to that of the spheroid luminosity $L_{s p h}$ versus $\sigma_{s p h}$. We note that up to half the scatter in this fundamental plane projection may be attributed to population age differences (Forbes, Ponman and Brown 1999) that affect primarily the mass-to-light ratios of spheroids. Another source of scatter that has also been discussed by Gebhardt et al. (2000a) is due to projection effects that lead to variations in the effective radius and velocity dispersion by a factor of order the typical ellipticity of the spheroid, up to a factor of 2 (Burkert and Naab 2000). The inferred intrinsic dispersion in the dependence of bulge mass, and also of black hole mass, on velocity dispersion must therefore have been quite small compared to the observed spread. Note that projection effects would result in scatter in the Magorrian relation as well as in scatter in the $M_{b h}-$ $\sigma_{\text {sph }}$ relationship. 
The following effect is likely to play an additional important role. Minor mergers add stars but should have little effect on the central black hole mass. These mergers could occur after the initial protospheroid formation phase, which characterises the formation of a central disk and the accretion of gas onto the central black hole but before the gas in the outer regions of the spheroid was completely consumed. The mergers induce a change in the star formation rate that is disproportionately larger than would be inferred directly from the amount of stars and gas added. This is because the tidal shocks stimulate the larger pre-existing outer gas supply to form stars earlier at an accelerated pace. Indeed the NUV-optical color-magnitude relation for early-type cluster galaxies is incompatible with a monolithic scenario for star formation at high redshift (Ferreras and Silk 2000). An increased scatter is found in the color-magnitude relation at the faint end, resulting in a significant fraction of faint blue early-type systems, implying that less massive galaxies undergo more recent episodes of star formation. Such episodes will produce scatter in the fundamental plane by affecting the galaxy luminosities and outer radii, but will not add appreciably to the scatter in the $\left(M_{b h}, \sigma_{s p h}\right)$ correlation because the velocity dispersion in the inner regions is not changed.

There is a further effect to be explained, namely the correlations between fundamental plane residuals (Kormendy 2000), specifically an anticorrelation between $\Delta \sigma_{s p h}$ and $\Delta r_{e}$. We attribute this as being in part due to projection effects of non-axisymmetric spheroids. In addition late mass loss or mass infall may well occur. Substantial mass loss or infall would modify both the galaxy size and spheroid velocity dispersion. In the limit of an adiabatic response of the host galaxy, we predict that

$$
\frac{\Delta r}{r}=-\frac{\Delta \sigma}{\sigma}=-0.4 \Delta M_{b}
$$

which is consistent with the observed anticorrelation beween $\Delta \sigma_{s p h}$ and $\Delta r_{e}$. The tightness of the $\left(M_{b h}, \sigma_{s p h}\right)$ correlation therefore means that late infall into or outflow from spheroids 
can be limited to about 10 percent of the current stellar mass. This complements a similar conclusion for disks, based on their observed thinness (Toth and Ostriker 1992).

Finally let us introduce cosmology via merger-induced feeding of the accretion disk. If the accretion disk lifetime exceeds the characteristic time between mergers, the gas inflow is disrupted. For example, a binary black hole merger would sweep out the local environment. The merger, by forming a transient bar, will subsequently resupply cold gas to the central object causing quasar activity. Whatever the details, it seems reasonable to equate the quasar lifetime $t_{q}=\gamma t_{e d d}$ to the merger time-scale $t_{\text {merger }}$, thereby introducing a dependence on the cosmological model. Here $\gamma$ (expected to be of order 0.1 ) is the ratio of $t_{q}$ to the Eddington time-scale $t_{e d d} \equiv 0.4 \mathrm{Gyr}$. Let $f_{\text {cold }}$ be the cold gas fraction of the total baryon content in the merging galaxy. One can then write

$$
t_{q} \equiv t_{\text {merger }}=\left(\frac{\bar{\sigma}_{s p h}}{\sigma_{0}}\right)^{\frac{3(3+n)}{1-n}} f_{\text {cold }}^{\frac{4}{1-n}} t_{0},
$$

where the normalization assumes that systems with velocity dispersion $\sigma_{0}=1000 \alpha \mathrm{km} \mathrm{s}^{-1}$, with $\alpha \approx 1$, are forming at the present epoch $t_{0}$. It follows that

$$
M_{b h}=\frac{t_{s f}}{t_{q}}\left(\frac{\sigma_{s p h}}{\sigma_{0}}\right)^{\frac{3(3+n)}{1-n}} f_{\text {cold }}^{\frac{4}{1-n}} t_{0} \frac{\sigma_{s p h}^{3}}{G R_{c r}} .
$$

The expected range for protogalaxies, $-1.5 \geq n \geq-2.5$, again yields the range of $4-5$ for the slope of the the $M_{b h}-\sigma_{s p h}$ relation.

Note that the expected narrow dispersion in $\sigma_{s p h}$ remains since most of the dependence on $\sigma_{s p h}$ (the part that varies as $\sigma_{s p h}^{3}$ ) is cosmology-independent. Note moreover, that there is no reliance on the uncertainties inherent in feedback, other than in the dependence on cold gas fraction, which may be adopted from the cold gas fraction for the observed damped Lyman alpha clouds. This varies approximately as $(1+z)^{-3 / 2}$ for $0<z<3$. We finally infer an approximately linear dependence of $M_{b h}$ on age of the stellar population, presumed to have formed when the cold gas fraction was supplied by the last major merger in accordance with observations. 


\section{Discussion}

A simple model of star formation-regulated black hole growth seems to explain the observed dependence of black hole on spheroid mass and spheroid velocity dispersion, and especially the observed dispersions. The proposed model leads to several predictions that might merit further investigation. We find that the Magorrian relation depends on two parameters, the critical viscous Reynolds number and the time-scale of star formation in units of the dynamical time-scale. As $\eta$ should be of order unity, we infer that black hole growth must be regulated by a turbulent accretion disk with a Reynolds number of a few

hundred. In addition, the accretion and star formation time-scales are closely coupled, and in our interpretation are of order the quasar lifetime.

The scatter of the Magorrian relation directly reflects the scatter in the fundamental plane parameters $r_{e}$ and $M_{b}$, which we postulate to be enhanced by secular events after black hole and spheroid formation. Thus the original scatter in the fundamental plane should be as small as that observed in the black hole mass-spheroid velocity dispersion relationship. This provides a possible observable prediction for high redshift field galaxies, and also for clusters where most of the merging may have been suppressed.

We wish to thank UCSC, UC Berkeley, IAP, Chez Papa and Cafe Peret for hospitality and for providing a stimulating atmosphere, 


\section{REFERENCES}

Adams, F., Graff and Richstone 2000, astro-ph/0010549.

Burkert, A. and Naab, T. 2000, in preparation.

Burkert, A. and Sunyaev, R. 2000, in preparation.

Duschl,W., Strittmatter,P and Biermann 2000, A\&A, 357,1123.

Ferrarese, L. and Merritt, D. 2000, ApJ, 539, 9.

Ferreras, I. and Silk, J. 2000, ApJ in press, astro-ph/0008493.

Forbes, D. A., Ponman, T. J. and Brown, R. J. N. 1998, ApJ, 508L, 43

Gebhardt, K. et al. 2000a, ApJ, 539L, 13.

Gebhardt, K. et al. 2000b, ApJ, 543L, 5.

Gnedin, O. and Ostriker, J. 2000, astro-ph/0010436.

Haehnelt, M. G. and Kauffmann, G. 2000, MNRAS, 318L, 35.

Haehnelt, M. G., Natarajan, P. and Rees, M. 1998, MNRAS, 300, 817.

Merritt, D. and Ferrarese, L. 2000, astr-ph/00090763.

Kormendy, J. 2000, Galaxy Disks and Disk Galaxies, ASP Conference Series, ed. J. Funes and E. Corsini, in press, astr-ph/0007401.

Magorrian, J. et al. 1998, AJ, 115, 2285.

Merrifield, M. R., Forbes, Duncan A. and Terlevich, A. I. 2000, MNRAS, 314, 768.

Mihos, C. and Hernquist, L. 1996, ApJ, 464, 641.

Ostriker, J. P. 2000, PRL, 84, 5258.

Toth, G. and Ostriker, J. P. 1992, ApJ, 389, 5.

Silk, J. and Rees, M. 1998, A\&A, 331L, 1. 
Toomre, A. and Toomre, J. 1972, ApJ, 178, 623.

Yoshida, N., Springel, V., White, S. and Tormen, G. 2000, ApJ, 535, L103. 